\title{
AN INVESTIGATION OF ACOUSTIC EMISSION SIGNAL ATTENUATION FOR MONITORING OF PROGRESSIVE FAILURE IN FIBERGLASS REINFORCED COMPOSITE LAMINATES
}

\author{
Z.M. Hafizi ${ }^{1,2}$, J. Epaarachchi ${ }^{1}$ and K.T. Lau ${ }^{1}$ \\ ${ }^{1}$ Centre of Excellence in Engineered Fibre Composites \\ Faculty of Engineering and Surveying \\ The University of Southern Queensland \\ Toowoomba, 4350, Australia \\ ${ }^{2}$ Faculty of Mechanical Engineering \\ Universiti Malaysia Pahang (UMP), Malaysia \\ Corresponding e-mail: mohdhafizibin.zohari@usq.edu.au
}

\begin{abstract}
Glass fiber reinforced polymer (GFRP) laminates have a high damping characteristic which can retard ultra-high frequency guided wave signals from propagating along the plate. Owing to this, the identification of an effective area for sensor location is essential. Appropriate signal processing, including wavelet analysis was done, in order to observe the details of an acoustic emission (AE) signal which was emitted at a distance from it source. This study reveals that it is necessary to consider the near field and far field effects of $\mathrm{AE}$ signal attenuation, in particular, to determine a composite's micro failure characterization. Consequently, this attenuation behavior was used to develop the non-velocity based source mapping to monitor the progressive failure in composite laminates. This paper details the outcomes of the signal energy attenuation method with an improved algorithm for progressive failure monitoring in fiberglass reinforced composite laminates and the 'effective length' for better AE detection.
\end{abstract}

Keywords: SHM; GFRP composite; acoustic emission; signal attenuation; wavelet analysis.

\section{INTRODUCTION}

Acoustic emission (AE) is a popular non-destructive technique (NDT) and its applications have increased substantially in structural health monitoring (SHM). During the past few decades, many successful research works have shown the remarkable capability of AE for early damage detection of composite materials (Mizutani et al., 2000; Eaton, Pullin, \& Holford, 2012; Trojanov et al., 2011). Due to the more complicated design and usage of composite materials in engineering structures nowadays, the evaluation method of $\mathrm{AE}$ for health monitoring also needs to be improved. Moreover, there is a significant challenge for a reliable AE based SHM system for composite structures due to the anisotropic nature of the materials. Much research has been undertaken to create more reliable quantitative AE for the SHM of composite structures (Scholey et al., 2010; Scholey et al., 2009). A recent study proved modal acoustic emission (MAE) to be the preferred method for source location detection and failure characterization. MAE has become the preferred method as it gives more accurate results than traditional $\mathrm{AE}$ has been shown to give. This concept fully utilizes the theory of wave propagation in solid media (e.g. beam, thin plates etc.) and 
signal processing such as spectral analysis and time-frequency analysis. MAE offers a better theoretical background for acoustic emission analysis, as it treats AE signals as mechanical waves which propagate through thin structures in a variety of modes. Plate wave theory and Lamb wave analysis for thin plates can clarify the existence of these wave modes in AE signals, as has been well explained in many previous works (Jeong, 2001; Jeong \& Jang, 2000; Prosser et al., 1995). According to Lamb's wave theory for thin plates, two major wave modes exist; extensional and flexural wave mode. The propagating waves have a dispersive characteristic, where different frequency components travel with different velocities (Lam et al., 2009), which can be presented through their dispersion curves. In a typical burst type AE waveform, the extensional wave mode usually propagates with higher velocity than the flexural mode (Prosser, 1991). Furthermore, the extensional wave mode can also be easily recognized by observation as its spectrum has a higher frequency component than the flexural mode. However, in high damping composites materials such as glass fiber resin polymer, higher frequency components may experience greater attenuation rates than the lower frequency components, which may lead to inaccurate information especially in the study of failure characterization. For instance, consider the case of matrix cracking in composite plates which was found to be dominated by the extensional mode (Scholey et al., 2010). If AE transducers are not placed at the appropriate distance from the source, the strength of the extensional waves might be attenuated and lost along their way to the sensors, thus losing the information. As far as the author is concerned, this is a gray area yet to be investigated. Research work on the optimal distance of the transducer from the crack is an essential requirement for the development of an $\mathrm{AE}$ based damage detection system.

Consequently the attenuation characteristics of AE signals can also be used as a location mapping tool. The accurate detection of a crack's location from a transducer significantly improves the ability to estimate crack propagation velocity and crack orientation. As such, location detection or damage localization in composite materials is a significant and developing research area. These research developments are motivated by the major drawbacks in the application of commercial AE systems for composite materials. There are many AE source location methods available for isotropic materials. The zonal method is one common AE source location finding method, but it uses a comparatively large number of sensors (Miller \& Hill, 2005; Prosser, 2002). Time of arrival (TOA) is another method which is frequently used for AE source location. It uses two AE sensors to locate an AE source in a linear direction and three sensors for a 2D plate. Most recently, an MAE-based source mapping method was developed [Jiao et al., 2006; Jiao et al., 2008] and the founders claimed that it produces improved accuracy. Furthermore, this technique makes single sensor linear source location detection possible (Jiao et al., 2004; Oskouei \& Ahmadi, 2008).

Most current source mapping techniques are dependent on the accurate measurement of wave velocity. Due to the varying wave velocity in composite materials, this phenomenon has not been properly investigated and remains an unsolved problem in the AE field. As a consequence, the next logical step is to investigate the use of non-velocity based approaches for AE source location detection. This paper details significant research work that has been conducted on AE source mapping techniques using the non-velocity based method. Fundamentally, the proposed method is based on an energy attenuation strategy in composite materials that is explained further in the next section. The modal study (MAE) of AE signals is also presented for a better understanding of effective area detection in high damping composite plates. 


\section{ANALYSIS}

\section{Modal Acoustic Emission}

In MAE, the knowledge of Lamb's wave theory for thin plates is necessary. In recent decades, many studies have utilized the Lamb wave theory for non-destructive testing (NDT) purposes, especially for ultrasonic inspection of thin plates (Lam et al., 2009; Tsuda, 2006; Diamanti \& Soutis, 2010). The Lamb wave can be used for NDT in two fields: first, in wave phase velocity, thickness calculation and elastic properties determination; and second in fault or damage detection (Diamanti \& Soutis, 2010)]. The dispersion curves of symmetric, $S$ and asymmetric, $A$ modes can be derived from Lamb's characteristic equation (Lam et al., 2009)

$$
\frac{\tan \left(\frac{\beta d}{2}\right)}{\tan \left(\frac{\alpha d}{2}\right)}=-\frac{4 \alpha \beta k^{2}}{\left(k^{2}-\beta^{2}\right)^{2}}
$$

for symmetric modes, and

$$
\frac{\tan \left(\frac{\beta d}{2}\right)}{\tan \left(\frac{\alpha d}{2}\right)}=-\frac{\left(k^{2}-\beta^{2}\right)^{2}}{4 \alpha \beta k^{2}}
$$

for asymmetric modes, where $\alpha=\sqrt{\left(\omega^{2} / c_{L}^{2}\right)-k^{2}}, \quad \beta=\sqrt{\left(\omega^{2} / c_{T}^{2}\right)-k^{2}}$ and $k=\omega / c_{p}$. The parameters $d, \omega, k, c_{p}, c_{L}$, and $c_{T}$, are the plate thickness, angular frequency, wave number, phase velocity, longitudinal wave velocity and transverse wave velocity respectively. Meanwhile, the relation between the group velocity, $c_{g}$ and phase velocity, $c_{p}$ can be expressed as (Lam et al., 2009)

$$
c_{g}=\frac{d k c_{p}}{d k}=c_{p}+k \frac{d c_{p}}{d k}
$$

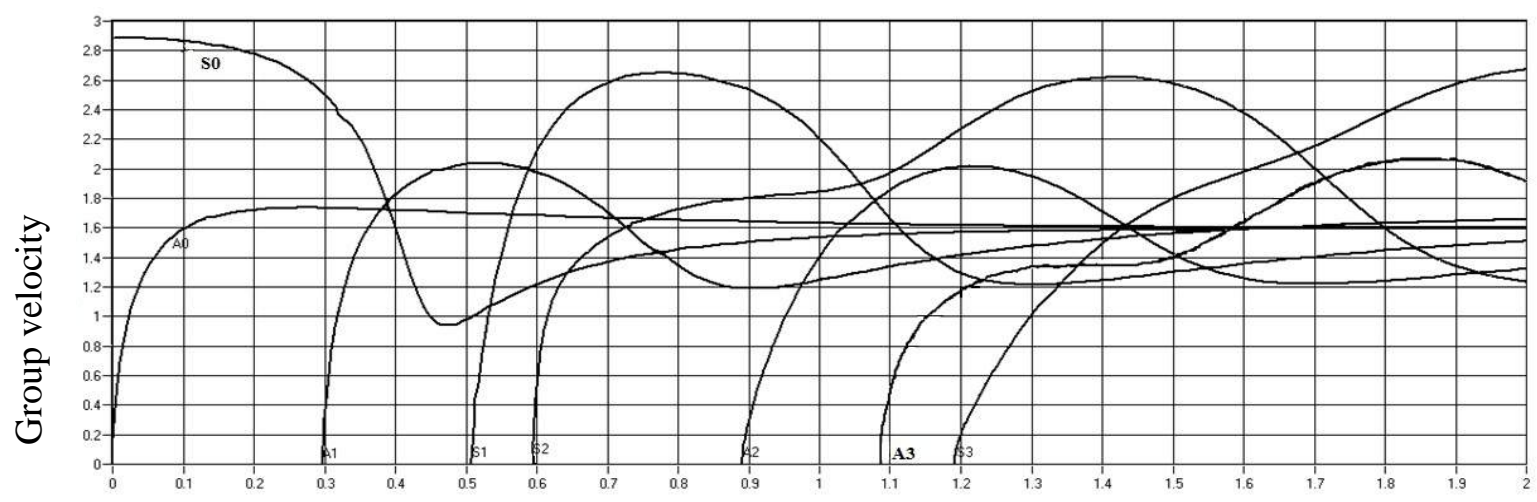

Frequency

Figure 1. Group velocity dispersion curves for the specimen.

An example of dispersion curves for glass fiber composite plates, which is calculated using the Lamb wave equation, is shown in Figure 1 (density $1600 \mathrm{~kg} / \mathrm{m}^{3}$ and 
thickness $2.9 \mathrm{~mm}$ ). Note that, for easier analysis, AE researchers were interested in a frequency range which contains only fundamental wave modes $S_{0}$ and $A_{0}$, which usually appear in a lower frequency range (Hafizi et al., 2012). For this purpose, an additional signal filtering process is required to ensure that only fundamental wave modes are captured by sensors. Besides the Lamb's wave analysis, MAE also requires the signal's time frequency information. For this particular purpose, continuous wavelet transform (CWT) provides better accuracy for both time and frequency information compared with the short-time Fourier transform (STFT) method (Daubechies, 1990). Several researchers interested in MAE have successfully used wavelet analysis, either for source location (Jiao et al., 2004; Jiao et al., 2006; Jiao et al., 2008; Oskouei et al., 2009; Hamstad et al., 2002) or for failure characterization (Ni \& Iwamoto, 2002; Marec et al., 2008). However, Hafizi et al. (2012) have shown the suitability of source mapping using STFT in their work.

The CWT of a function, as defined by Chui (1992), can be expressed as

$$
W T_{f}(s, \tau)=\frac{1}{\sqrt{s}} \int_{-\infty}^{\infty} f(t) \psi^{*}\left(\frac{t-\tau}{s}\right) d t
$$

where $s>0$ and the superscript * indicates the complex conjugate. The term $\psi(t)$ is the basic wavelet. The parameter $s$ in Eq. (4) stands for the scale of the basic wavelet and is related to signal frequency. Meanwhile, the parameter $\tau$ stands for the shift or position of the basic wavelet and it can be related to the time of the signal. Plotting the wavelet transform magnitude on the $s-\tau$ axis gives the time-frequency view of a signal with improved time and frequency resolution compared to STFT.

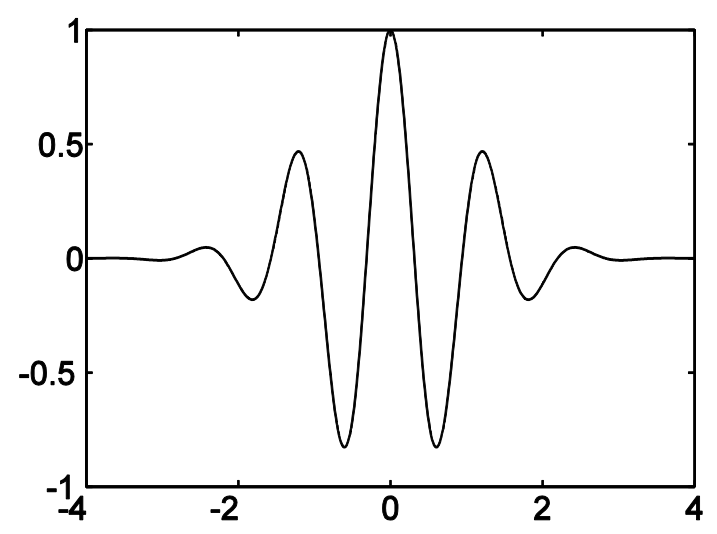

Figure 2. The shape of the Morlet wavelet.

There are many basic wavelets available and the appropriate wavelet choice will give better results. In this study, the wavelet used was the Morlet wavelet (as shown in Figure 2) which is identical to the Gabor wavelet (Simonovski \& Boltezar, 2003) and has a similar shape to an impulse (Lin, 2001). It can be defined as (Lin, 2001; Simonovski \& Boltezar, 2003)

$$
\psi(t)=\left(e^{-i \omega_{0} t}-e^{-\omega^{2} / 2}\right) e^{-t^{2} / 2}
$$

The scale, $s$ can be related to the frequency by this relation: 


$$
\omega=\eta / s
$$

where the coefficient $\eta$ can also be written as the wavelet center frequency, $\omega_{0}$ depending on the sampling frequency and the selected minimum scale, as explained by Simonovski and Boltezar (2003).

\section{Signal Energy Equation}

Generally, AE waves propagate in the ultrasonic range; and in an ultrasonic wave study, it is necessary to include the effect of wave signal attenuation. In general, the sound wave attenuates with this relation (Shull \& Tittmann, 2002),

$$
A=A_{0} e^{-\alpha z}
$$

where $A_{0}$ is the amplitude of the propagating wave at the initial point or defect source. The amplitude $A$ is the decreased amplitude as the wave travels in the distance $z$ from the source or initial location. The $\alpha$ value refers to the attenuation coefficient of the wave traveling in the $z$-direction, and the dimension can be written as nepers/length.

The same model can be applied for the signal energy attenuation of AE signals propagation on thin composite plates, which can be expressed as a universal model:

$$
E_{i}=E_{0} e^{-\beta\left(\left|x_{i}-x_{0}\right|\right)}
$$

where $x_{0}$ refers to the location of the source, $x_{i}$ is the location of sensor $i, E_{0}$ is the energy at the source, $E_{i}$ is energy at sensor $i$ and $\beta$ is the decay constant. Equation (8) can be used for linear source location detection using a set of two sensors. In the case of value $\beta$ being unknown, three sensors are needed and it can be expressed as

$$
\left(\ln \frac{E_{3}}{E_{2}} / \ln \frac{E_{2}}{E_{1}}\right)=\frac{\left|x_{2}-x_{0}\right|-\left|x_{3}-x_{0}\right|}{\left|x_{1}-x_{0}\right|-\left|x_{2}-x_{0}\right|}
$$

\section{EXPERIMENTATION}

Three test setups were designed and prepared for signal energy attenuation, non-velocity source mapping and effective detection length study. For this purpose, $400 \mathrm{~mm} \times 400$ $\mathrm{mm} \times 2.9 \mathrm{~mm} \mathrm{GI} / \mathrm{epoxy}$ resin laminates with a stacking sequence $\left[0^{\circ}\right]_{4}$ were fabricated using the hand lay-up method. The plates' signal energy attenuation rates were plotted for several different angles; $0^{\circ}, 45^{\circ}, 60^{\circ}$ and $90^{\circ}$, by breaking the pencil lead at several points along the given angles in a straight line, starting from one edge of the composite specimen towards the other edge. As an example, Figure 3 shows the setup for the $45^{\circ}$ test. Note that the angle $0^{\circ}$ refers to a line along the glass fiber. For data acquisition, two single-channel Physical Acoustic Corporation (PAC) AE Node Systems were used. Each of them was connected with two different types of AE sensors (from PAC) which were NANO-30 with working frequency $100 \mathrm{kHz}$ to $700 \mathrm{kHz}$ (channel 1) and $R 6 \alpha$ with resonance frequency $60 \mathrm{kHz}$ (channel 2).

In the meantime, AE Win software was used to control the acquisition process. For instance, all the acquired signals were filtered in the range of $20 \mathrm{kHz}$ to $1000 \mathrm{kHz}$ 
because the fundamental wave propagation mode was targeted in this study. The sampling rate for acquisition was set up to 5 Mega samples per second and the threshold was set to $45 \mathrm{~dB}$.

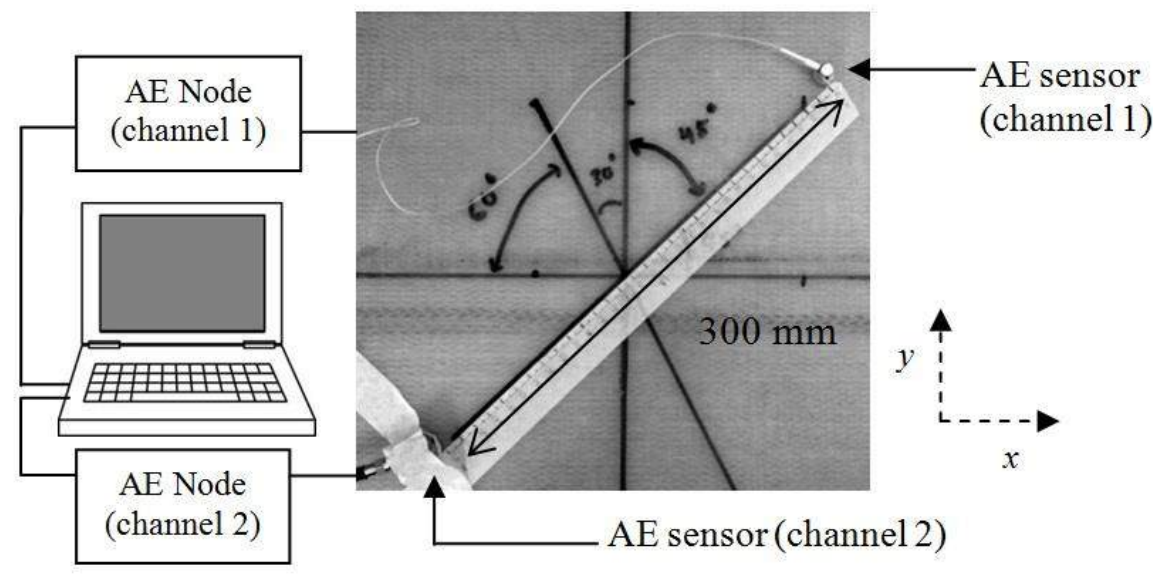

Figure 3. Arrangement for attenuation rate study.

As a validation of the non-velocity source mapping approach, a simple test was done by applying a pencil lead break as an artificial AE source at several locations on the specimen. For each location, AE signals were acquired at three places (A, B and C) as indicated in Figure 4. Note that only one NANO-30 sensor and a single-channel AE node for data acquisition was used. This procedure was repeated for angles $0^{\circ}, 45^{\circ}, 60^{\circ}$ and $90^{\circ}$. All signals were recorded and the linear source mapping was determined using Equation 9, as explained in the next section.

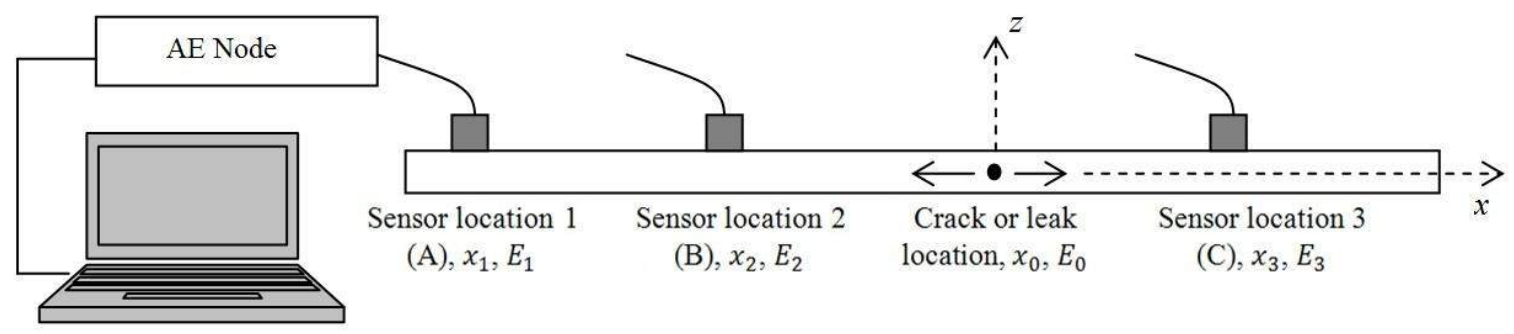

Figure 4. Diagram for non-velocity source mapping study.

To study the effective length of $\mathrm{AE}$ detection, the same specimen was cut into a smaller size: $250 \mathrm{~mm} \times 25 \mathrm{~mm}$ (250 $\mathrm{mm}$ length is in the fiber direction). Four singlechannel AE Node systems were combined to create a four-channel AE system for data acquisition, and the full setup was as shown in Figure 5. All AE Nodes were set to sample AE data at $1 \mathrm{Mega}$ sample per second. One end of the specimen was clamped to a support, while the other end was screwed to a shaker to form a simply supported beam condition. Four AE sensors $(R 6 \alpha)$ were attached to the sample (numbered as in Figure 5 ) and the pencil lead break test was done to the right of the sensor labeled "1". Note that sensor 4 was located $205 \mathrm{~mm}$ from the shaker. Later, a $100 \mathrm{kHz}$ vibration was applied to the specimen through the shaker. 


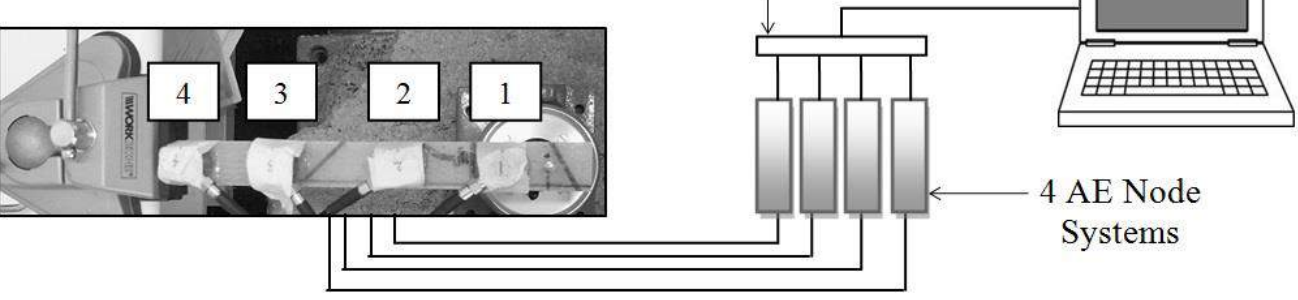

Figure 5. Setup for effective length study.

\section{RESULTS AND DISCUSSION}

Two types of AE sensor were used in this work: broadband, NANO-30 and resonance frequency sensor, $R 6 \alpha$. Figure 6 shows the resulting comparison of $\mathrm{AE}$ signal capture for each sensor with the same AE source (pencil lead break). With respect to the MAE study, a broadband sensor is better due to its ability to receive more frequency content from the AE source, as seen in Figure 6(c) and (d), therefore giving accurate information for composites' early failure characterization. However, the total signal energy that was acquired from the broadband sensor was far less than the total energy of AE signals obtained through the resonance frequency sensor. Although the total energy was different, it didn't affect the attenuation pattern of the AE signals' energy, as indicated in Figure 7.
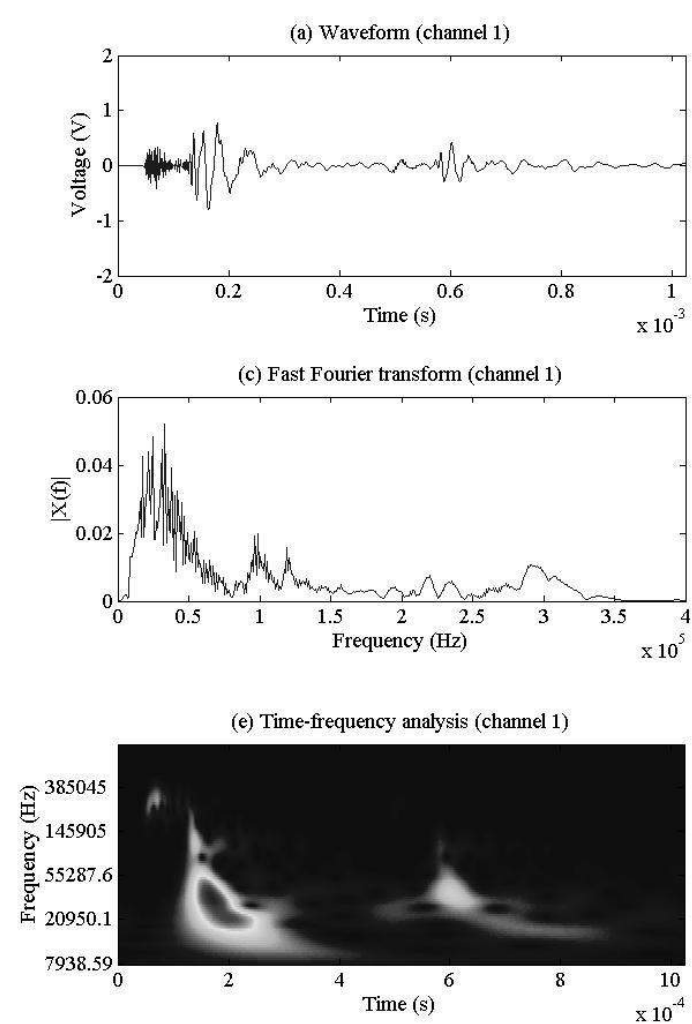
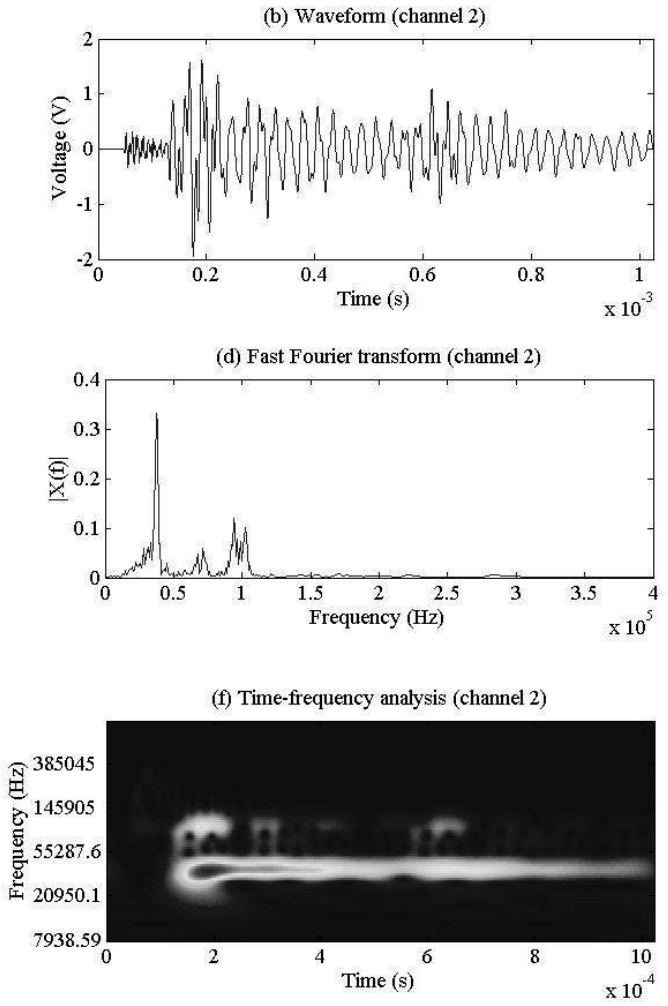

Figure 6. Comparison of AE signals captured by broadband and resonance frequency sensor. 

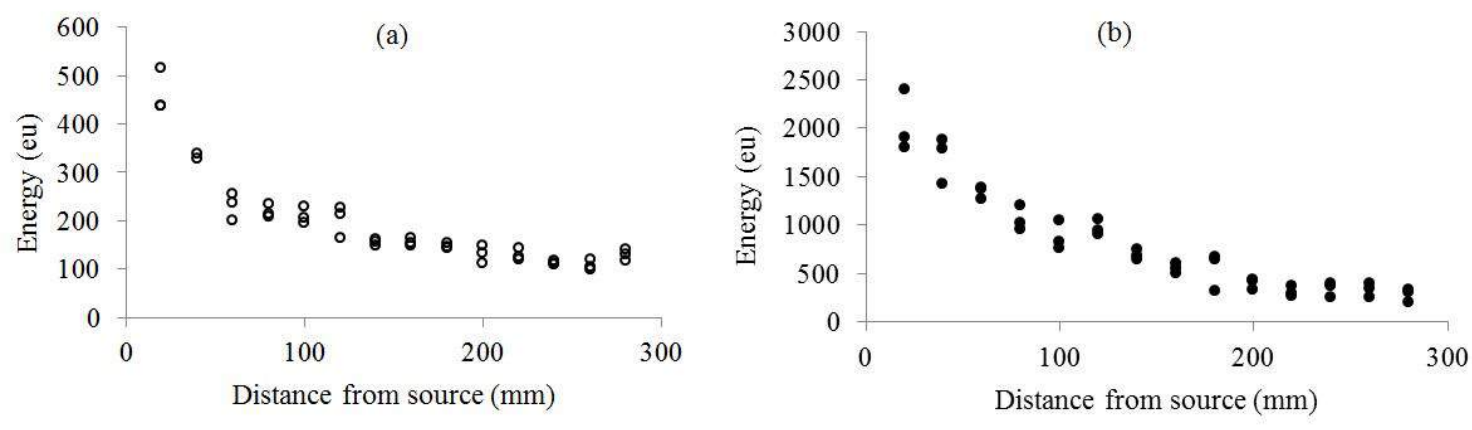

Figure 7. Energy attenuation of AE signals using (a) broadband sensor and (b) resonance frequency sensor for test angle $0^{\circ}$.

\section{Non-Velocity Based Source Mapping}

Figure 8 illustrates the decay rate of the AE signals' energy in the composite specimen for four different test angles. It can be observed that the AE signals' energy attenuation for the specimen follows the general model of ultrasonic wave attenuation; the exponential pattern is as Eq. (7), regardless of the test angles used. The R-square showed quite high values and the average decay constant for all test angles was 0.006. However, a new attenuation pattern can be proposed from the result in Figure 8: a logarithmic pattern. The dotted line in Figure 8 shows the logarithmic trend line, and the $\mathrm{R}$-square values were increased for all angles. Based on this, a new universal model for signal energy attenuation for the source location detection algorithm is proposed:

$$
E_{i}=\ln \left(x_{i}-x_{0}\right)^{-\gamma}+E_{0} \quad ; x_{0} \neq x_{i}
$$

where $\gamma$ is the logarithmic decay constant. For three sets of linearly arranged transducers, the equation can be expressed as

$$
\frac{\left(E_{3}-E_{2}\right)}{\left(E_{2}-E_{1}\right)}=\frac{\ln \left(\left|x_{2}-x_{0}\right| /\left|x_{3}-x_{0}\right|\right)}{\ln \left(\left|x_{1}-x_{0}\right| /\left|x_{2}-x_{0}\right|\right)}
$$

or can be written as

$$
\left(\left|x_{1}-x_{0}\right|\right)^{\Gamma}\left(\left|x_{3}-x_{0}\right|\right)=\left(\left|x_{2}-x_{0}\right|\right)^{(1+\Gamma)}
$$

where $\Gamma=\left(E_{3}-E_{2}\right) /\left(E_{2}-E_{1}\right)$. Equation (12) was used as an improved non-velocity based linear source localization. Note that, since the limitation of this proposed algorithm is $x_{0} \neq x_{i}$, the sensor location must not be located very close to the expected defect area. 
(a)

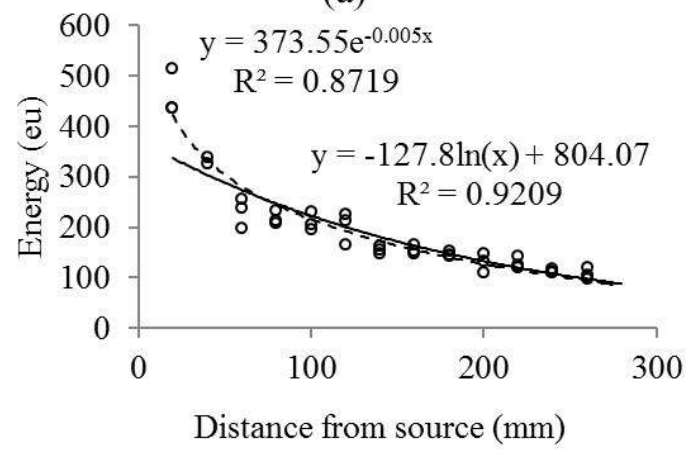

(c)

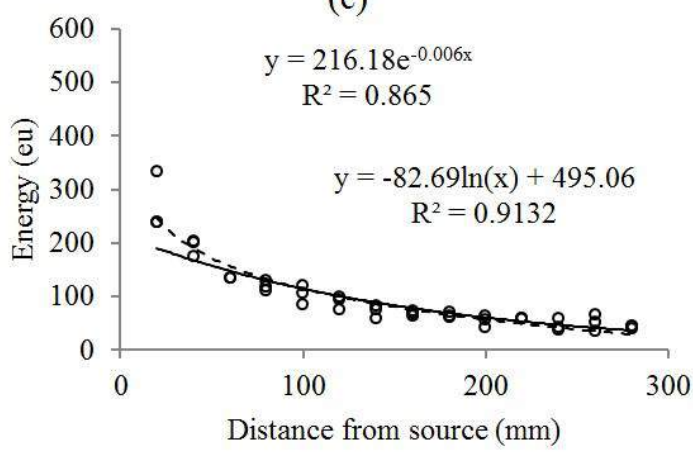

(b)

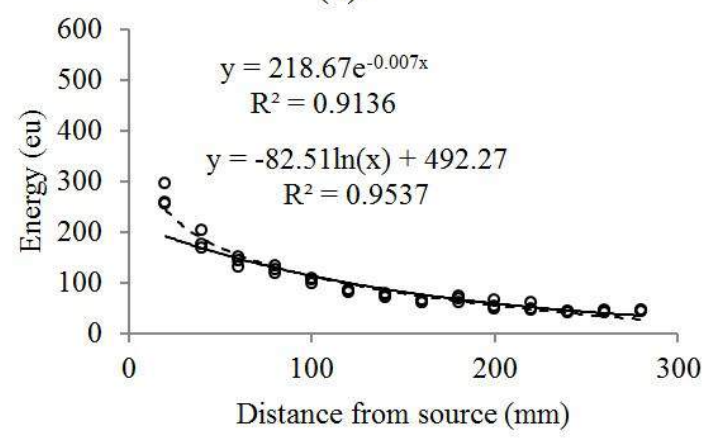

(d)

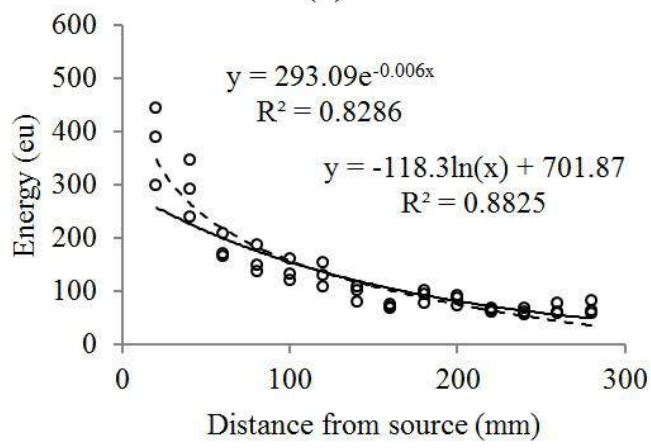

Figure 8. (a) Attenuation rate for $0^{\circ}$, (b) attenuation rate for $45^{\circ}$, (c) attenuation rate for, $60^{\circ}$ and (d) attenuation rate for $90^{\circ}$. The solid lines are the exponential trend line while the dotted lines are the logarithmic trend line.

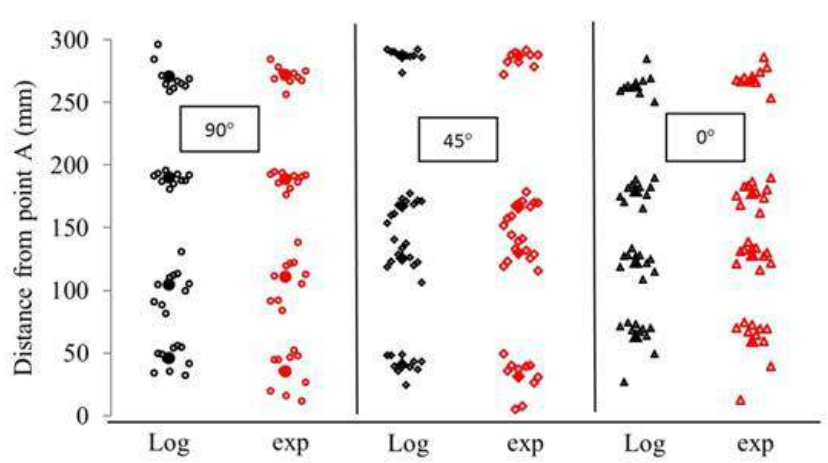

(a)

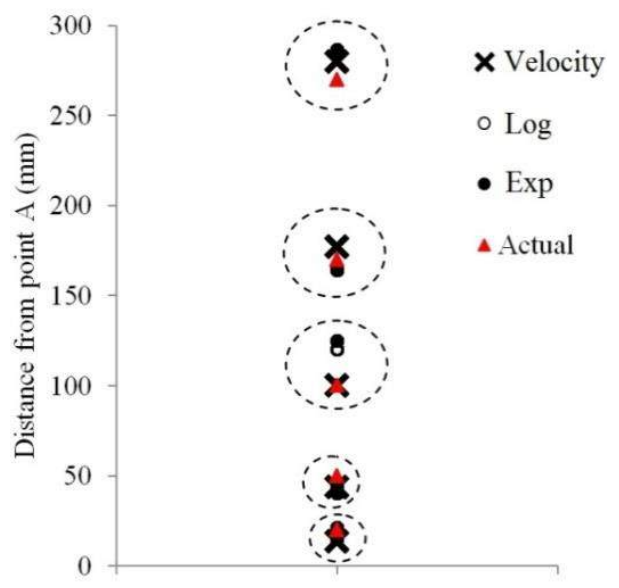

(b)

Figure 9. (a) Results comparison between exponential [Exp] and logarithmic attenuation model [Log]; (b) Comparison between logarithmic model [Ln], exponential model [e], actual location and commercial AE tool [velocity].

The results for the source mapping validation are shown in Figure 9 (a). All the plots are randomly scattered very close to the actual source for both models: exponential and logarithmic. Although they do not look much different, it appears that the 
logarithmic model is more stable and spotted very closely at the same average value compared to the exponential model. Furthermore, when the source was near to points A and $\mathrm{C}$, the exponential model calculated the source location with a huge error and a correction was required in order to find the accurate location. On the other hand, this problem didn't occur when calculating using the logarithmic model. The errors for both models compared with the actual location are summarized in Table 1. In the meantime, Figure 9 (b) reveals the average value for the logarithmic and exponential models, compared with the actual source location and source location using commercial AE tools for test angle $45^{\circ}$.

Table 1. Error comparison between logarithmic and exponential source location model.

\begin{tabular}{cccccc}
\hline & Log $(\mathrm{mm})$ & Exp $(\mathrm{mm})$ & Actual $(\mathrm{mm})$ & $\begin{array}{c}\text { Log error } \\
(\mathrm{mm})\end{array}$ & $\begin{array}{c}\text { Exp error } \\
(\mathrm{mm})\end{array}$ \\
\hline \multirow{4}{*}{$0^{\circ}$} & 45.65 & 34.97 & 50.00 & 4.35 & 15.03 \\
& 104.10 & 110.37 & 100.00 & 4.10 & 10.37 \\
& 189.67 & 188.91 & 170.00 & 19.67 & 18.91 \\
& 270.44 & 271.72 & 270.00 & 0.44 & 1.72 \\
\hline \multirow{4}{*}{$45^{\circ}$} & 44.42 & 36.96 & 50.00 & 5.58 & 13.04 \\
& 120.23 & 125.27 & 100.00 & 20.23 & 25.27 \\
& 165.73 & 163.78 & 170.00 & 4.27 & 6.22 \\
$90^{\circ}$ & 284.42 & 283.58 & 270.00 & 14.42 & 13.58 \\
\hline & 123.53 & 59.98 & 50.00 & 13.53 & 9.98 \\
& 179.68 & 128.66 & 100.00 & 23.38 & 28.66 \\
& 263.96 & 269.90 & 270.00 & 6.04 & 8.28 \\
\hline
\end{tabular}

\section{Effective Detection Area}

Although there are big differences between the AE and acoustics studies, especially in term of their applications (Loman, 2013), they actually share a few characteristics. In acoustics, the area surrounding a sound source can be divided into different fields; e.g. near field and far field (Hansen, 2013). Due to the attenuation behavior of sound pressure, the near field region can be limited from the source to the distance equal to one third of the largest energy or amplitude of the sound source. This term can be useful when relating the results presented in the previous section with the effective detection area. Figure 10 reveals the detailed analysis of an AE signal which was obtained at 5 different distances from the AE source. A single AE signal may contain a reasonably large amount of information from the source, particularly due to micro-failure formation and its propagation. The frequency spectrum of the signal was proved to be one of the important parameters that indicate the type of micro-failure characteristic (Gutkin et al., 2011). As explained earlier, the extensional wave mode conventionally has faster velocity, higher frequency and lower amplitude (due to inplane motion) than the flexural mode. Referring to the wavelet analysis as shown in Figure $10(\mathrm{k}, 1, \mathrm{~m}, \mathrm{n}, \mathrm{o})$, the extensional mode 'signature' was consistent with the 300 $\mathrm{kHz}$ component which can be seen fading as the distance from the AE source increases. In other words, the extensional mode almost disappears at the far field, approximately $140 \mathrm{~mm}$ from the source (see Figure 7a). In practice, this will affect the accuracy of 
information for micro-failure characterization since, in the MAE study, different failure mechanisms or source orientations can generate different frequency or wave propagation modes. Meanwhile, the flexural wave mode ranges from $20 \mathrm{kHz}$ to 250 $\mathrm{kHz}$. They were seen arriving at the sensor later than the extensional mode and became slower as the distance from the $\mathrm{AE}$ source increased; this is consistent with antisymmetric mode behavior. Note that, at $260 \mathrm{~mm}$ distance from the AE source, only the lowest frequency component of the flexural wave was left.
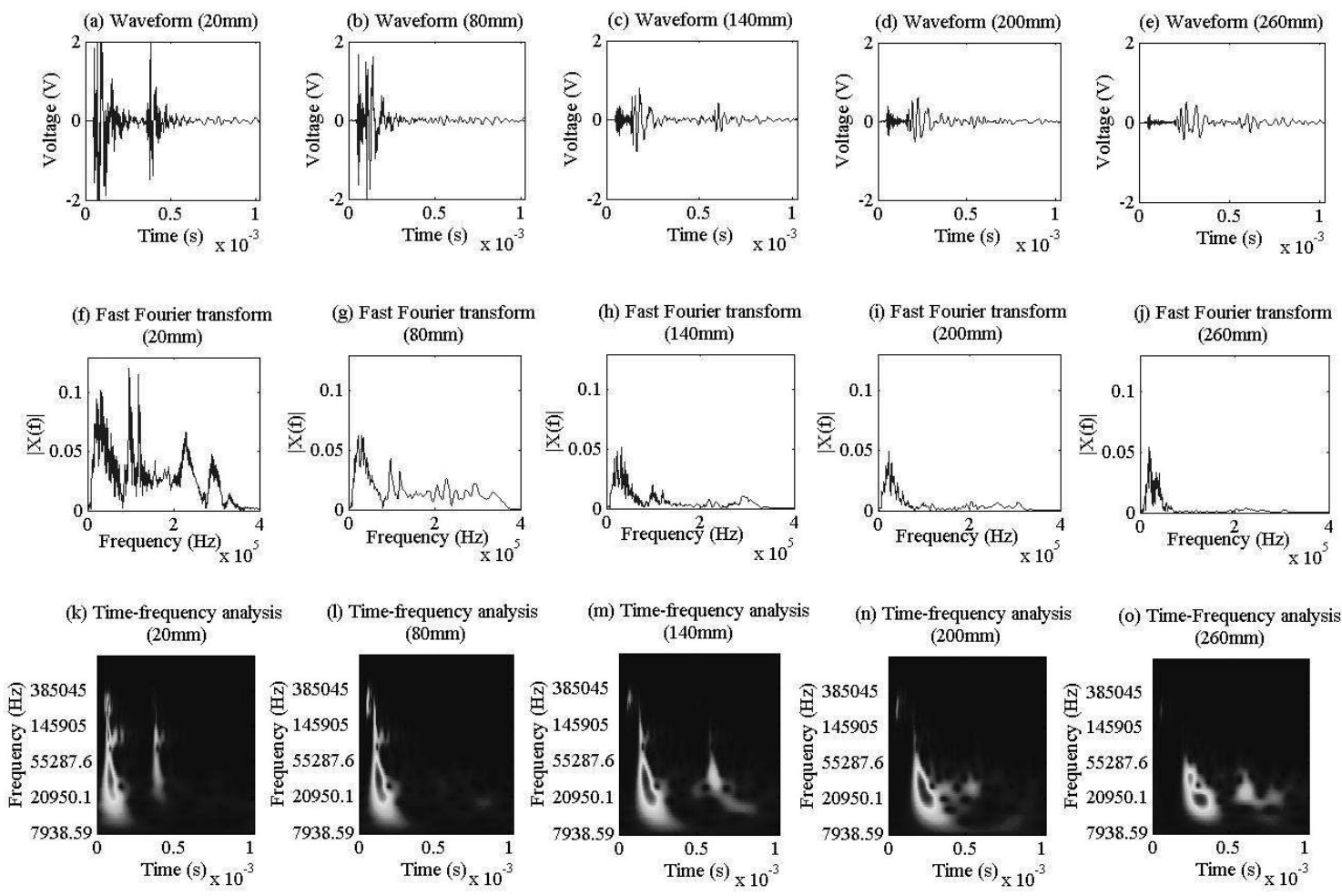

Figure 10. Detailed analysis of AE signals obtained from 5 different distances from AE source at test angles $0^{\circ}$.

The pencil lead break test results shown in Figure 11 (a to h) also reveal the same pattern and can be used to explain the effective area for better AE signal detection. The results corresponded with the setup as in Figure 5. Two main frequency components exist: high frequency (approx. $100 \mathrm{kHz}$ ) and low frequency (approx. $30 \mathrm{kHz}$ ). The signal from sensor 4 (Figure 11d) had the lowest energy/RMS value and the distance from the AE source was $205 \mathrm{~mm}$. By comparing the frequency spectrum obtained from signal 1 (Figure 11e) and signal 4 (Figure 11h), it appeared that the amplitude of the higher frequency component dropped faster, especially after $205 \mathrm{~mm}$ distance from the AE source. 

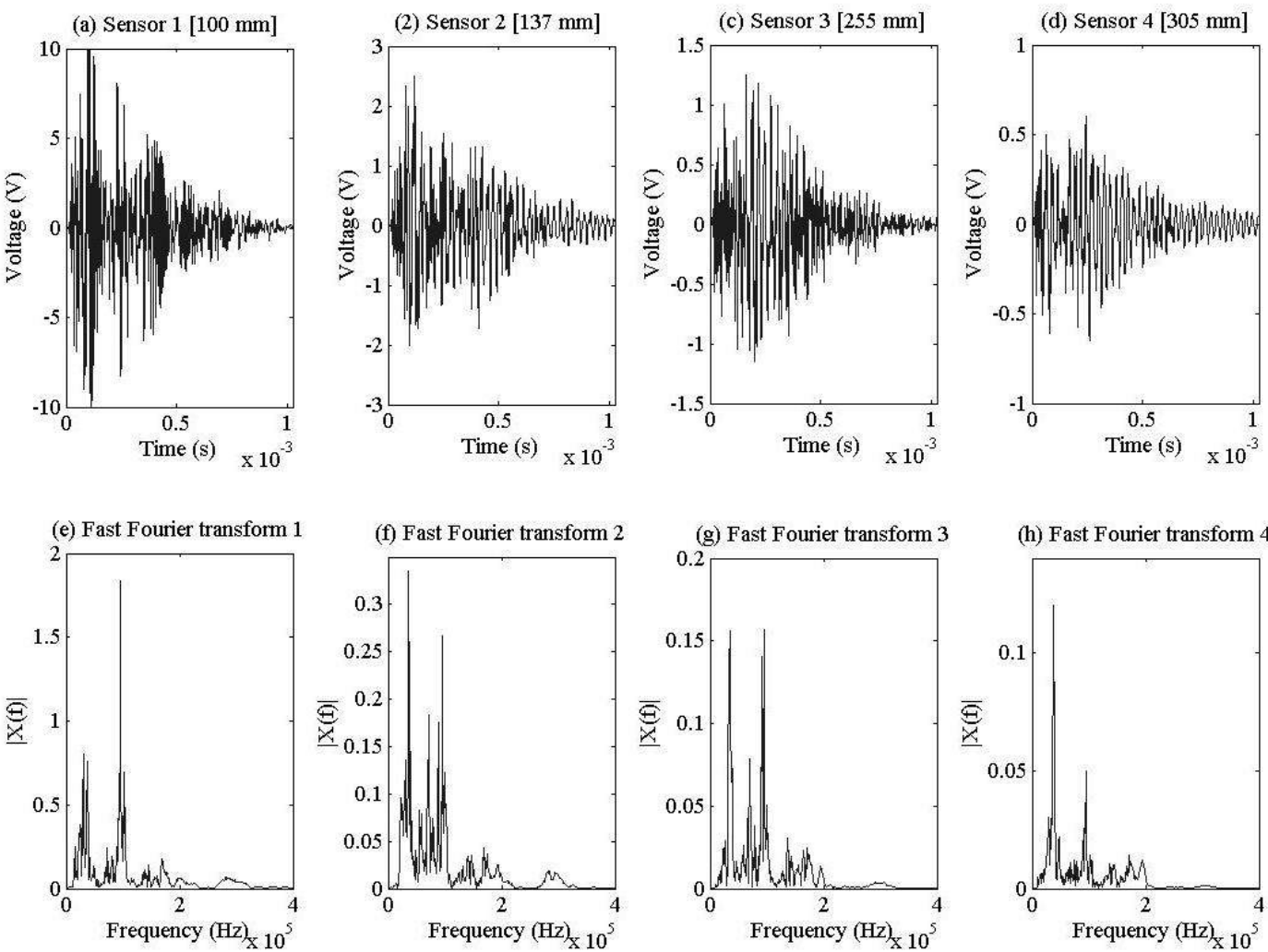

Figure 11. (a) AE signal from sensor 1; (b) AE signal from sensor 2; (c) AE signal from sensor 3; (d) AE signal from sensor 4; (e - h) Fast Fourier Transform [FFT] for AE signals in $(\mathrm{a}-\mathrm{d})$ respectively.

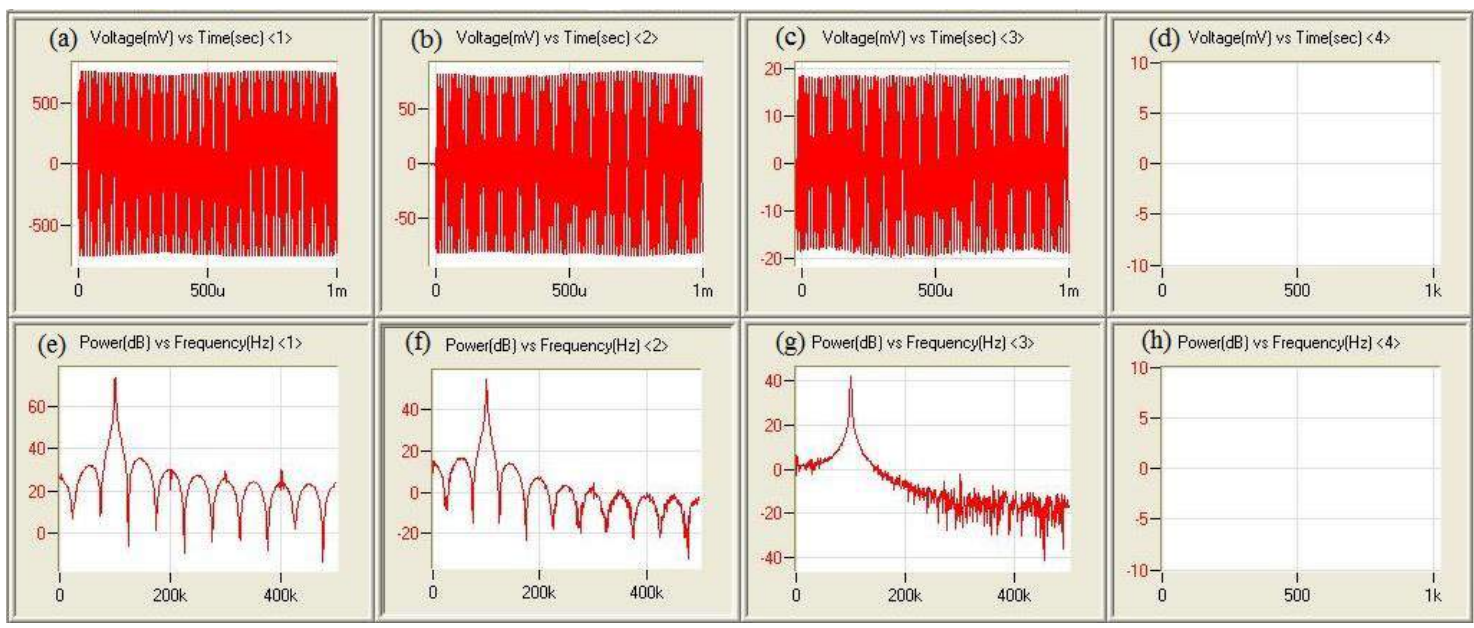

Figure 12. Result with $100 \mathrm{kHz}$ vibration applied to the specimen.

Obviously, within a short distance, the propagating AE signal was dominated by a low frequency signal, even though it was earlier dominated by a high frequency signal. This 'turning-over' starts approximately in between sensors 2 and 3, or $120 \mathrm{~mm}$ from the AE source, which is at the limit of the near field region (see Figure $7 \mathrm{~b}$ ). It is clear that a frequency of more than $100 \mathrm{kHz}$ was difficult to detect within a $205 \mathrm{~mm}$ radius 
(at sensor number 4), as indicated in Figure 12. At the same time, failures such as delamination and fiber breakage are reported to create AE signals at more than $100 \mathrm{kHz}$ (Gutkin et al., 2011)]. This explains how significant the effective detection area is as an important criterion for creating improved SHM for composite materials.

\section{CONCLUSIONS}

The study successfully presented the effective length detection for AE signals in a fiberglass reinforced epoxy resin composite. It is recommended that any AE transducers must be attached or embedded approximately $200 \mathrm{~mm}$ from the expected damage area, or the near field region, to avoid any important AE signal information being diminished due to attenuation and dispersion. The study also concluded that the non-velocity dependent source location algorithm utilizing both the exponential model and the logarithmic model shows a promising approach to damage localization for composite materials. Note that a linear mapping technique was presented. However, for 2D planar source location, the concept of energy attenuation source location can also be applied with an appropriate number of sensors.

\section{ACKNOWLEDGMENTS}

The authors would like to thank the Faculty of Engineering and Surveying and the Centre of Excellence in Engineered Fibre Composites, University of Southern Queensland for providing laboratory facilities and financial support. They would also like to thank the Universiti Malaysia Pahang together with the Ministry of Higher Education, Malaysia for scholarship support.

\section{REFERENCES}

Chui, C. K. (1992). An introduction to wavelets. San Diego, CA: Academic Press Professional.

Daubechies, I. (1990). The wavelet transform, time frequency localization and signal analysis. IEEE Transactions on Information Theory, 36(5), 961-1005.

Diamanti, K. \& Soutis, G. (2010). Structural health monitoring techniques for aircraft composites structures. Progress in Aerospace Sciences, 46, 342-352.

Eaton, M. J., Pullin, R., \& Holford K. M. (2012).Acoustic emission source location in composite materials using delta t mapping. Composites: Part A, 43, 856-863.

Gutkin, R., Green, C. J., Vangrattanachai, S., Pinho, S. T., Robinson P., \& Curtis, P. T. (2011). On acoustic emission for failure investigation in CFRP: Pattern recognition and peak frequency analyses. Mechanical Systems and Signal Processing, 25, 1393-1407.

Hafizi, Z. M., Epaarachchi, J., \& Lau, K. T. (2012). Modal analysis of high frequency acoustic signal approach for progressive failure monitoring in thin composite plates. In MPC 2012: 2nd Malaysian Postgraduate Conference, Gold Coast, Australia, 7-9 July.

Hamstad, M. A., O'Gallagher, A., \& Gary, J. (2002). A wavelet transform applied to acoustic emission signals part 2: Source location. Journal of Acoustic Emission, 20,62-82.

Hansen, C. H. (2013). Fundamentals of acoustics. World Health Organization, viewed 5 Feb. 2013, <http://www.who.int/occupational_health/publications/noise1.pdf>. 
Jeong, H. (2001). Analysis of plate wave propagation in anisotropic laminates using a wavelet transform. NDT \& E International, 34, 185-190.

Jeong, H. \& Jang, Y. S. (2000). Wavelet analysis of plate wave propagation in composite laminates. Composites Structures, 49, 443-450.

Jiao, J.-P., He, C.-F, Wu, B., Fie, R.-Y., \& Wang, X.-Y. (2006). A new acoustic emission source location technique based on wavelet transform and mode analysis. Frontiers of Mechanical Engineering China, 3, 341-345.

Jiao, J.-P., Wu, B., \& He, C.-F. (2008). Acoustic emission source location methods using mode and frequency analysis. Structural Control and Health Monitoring, 15, 642-651.

Jiao, J.-P., He, C.-F., Wu, B., Fei, R.-Y., \& Wang, X.-Y. (2004). Application of wavelet transform on modal acoustic emission source location in thin plates with one sensor. International Journal of Pressure Vessels and Piping, 81, 427-431.

Lam, P. M, Lau, K. T., Ling, H. Y., Su, Z., \& Tam, H. Y. (2009). Acousto-ultrasonic sensing for delaminated GFRP composites using an embedded FBG sensor. Optics and Lasers in Engineering, 47, 1049-1055.

Lin, J. (2001). Feature extraction of machine sound using wavelet and its application in fault diagnosis. NDT \&E International, 34, 25-30.

Loman, M. (2013). Between acoustics and acoustic emission; a justification. Advanced Structural Integrity and Vibration Research, Universiti Malaysia Pahang, Malaysia, viewed 3 Feb. 2013, < http://asivr.ump.edu.my/>.

Marec, A., Thomas, J. H., \& Guerjouma, R. E. (2008). Damage characterization of polymer-based composite materials: Multivariable analysis and wavelet transform for clustering acoustic emission data. Mechanical Systems and Signal Processing, $22,1441-1464$.

Miller, R. K. \& Hill, E. V. K. Nondestructive testing handbook: Acoustic emission testing, volume 6 ( $3^{\text {rd }}$ ed.). American Society for Nondestructive Testing Inc., USA.

Mizutani, Y., Nagashima, K., Takemoto, M., \& Ono, K. (2000). Fracture mechanism characterization of cross-ply carbon-fiber composites using acoustic emission analysis. NDT \& E International, 33, 101-110.

Ni, Q. Q. \& Iwamoto, M. (2002). Wavelet transform of acoustic signals in failure of model composites. Engineering Fracture Mechanics, 69(6), 717-728.

Oskouei, A. R. \& Ahmadi, M. (2008). Using wavelet transform to locate acoustic emission source in composite plate with one sensor. In Acoustics '08, Paris, France, 29 June- 4 July.

Oskouei, A. R., Ahmadi, M., \& Hajikhani, M. (2009). Wavelet-based acoustic emission characterization of damage mechanism in composite materials under mode I delamination at different interfaces. Express Polymer Letters, 3(12), 804-813.

Prosser, W. H., Jackson, K. E., Kellas, S., Smith, B. T., McKeon, J., \& Friedman, A. (1995). Advanced waveform based acoustic emission detection of matrix cracking in composites. Materials Evaluation, 53 (9), 1052-1058.

Prosser, W. H. (1991). The propagation characteristic of the plate modes of acoustic emission waves in thin aluminium plates and thin graphitelepoxy composite plates and tubes (PhD thesis). The Johns Hopkins University, 1991.

Prosser, W. H. (2002). Nondestructive evaluation: Theory, techniques and applications, volume 6 (Acoustic Emission). New York: Marcel Dekker. 
Scholey, J. J., Wilcox, P. D., Wisnom, M. R., \& Friswell, M. I. (2010). Quantitative experimental measurements of matrix cracking and delamination using acoustic emission. Composites: Part A, 41, 612-623.

Scholey, J. J., Wilcox, P. D., Wisnom, M. R., \& Friswell, M. I. (2009). A practical technique for quantifying the performance of acoustic emission systems on platelike structures. Ultrasonics, 49, 538-548.

Shull, P. J. \& Tittmann, B. R. (2002). Nondestructive evaluation: Theory, techniques and applications, volume 3 (Ultrasound). New York: Marcel Dekker.

Simonovski, I. \& Boltezar, M. (2003). The norms and variances of the Gabor, Morlet and general harmonic wavelet functions. Journal of Sound and Vibration, 264, 545-557.

Trojanov, Z., Szraz, Z., Chmelk, F., \& Lukc, P. (2011) Acoustic emission from deformed magnesium alloy based composites. Materials Science and Engineering A, 528(6), 2479-2483.

Tsuda, H. (2006). Ultrasound and damage detection in CFRP using fiber Bragg grating sensors. Composites Science and Technology, 66, 676-683. 